\title{
La comunicación, interacción y entendimiento en la Europa multicultural a través de la actualización de los programas de formación del profesorado especialista en lengua extranjera
}

Communication, interaction and understanding in multicultural Europe through an update of foreign language teacher training programs

\author{
Gabriel Sánchez Sánchez ${ }^{1}$ \\ gsanchezsanchez@um.es \\ Universidad de Murcia, España
}

\section{Resumen:}

La consolidación de Europa como una unidad territorial tras la II Guerra Mundial condiciona la aparición de un trasfondo multilingüístico y multicultural sin precedentes. La política lingüística del Consejo de Europa estará enfocada principalmente a garantizar la comunicación, el enriquecimiento, la comprensión y la cohesión territorial y social entre los estados de la unión. Este objetivo origina toda una serie de necesidades y exigencias a las que se habrá que dar respuesta desde las Administraciones educativas. La labor de estas consiste en continuar la trayectoria iniciada por el Consejo de Europa y establecer los objetivos que deben plantearse las instituciones educativas para contribuir de ese modo al logro de ese entendimiento

\begin{abstract}
:
The consolidation of Europe as a territorial unit after the World War II determines the appearance of a multilingual and multicultural unprecedented background. The linguistic policy designed by the Council of Europe will focus mainly on ensuring the communication, enrichment, understanding and territorial and social cohesion between the states of the European Union. This objective creates some needs and requirements which will have to be satisfied by the Educational Authorities. These organizations are expected to continue the path initiated by the Council of Europe and establish the objectives that educational institutions are to meet to contribute to the achievement of this European understanding and cohesion. As a result of this,
\end{abstract}

1 Dirección para correspondencia (correspondence address):

Gabriel Sánchez Sánchez. Dpto. de Didáctica de la Lengua y la Literatura. Facultad de Educación. Universidad de Murcia. Campus de Espinardo, s/n. 30100 Murcia (España). 
La comunicación, interacción y entendimiento en la Europa multicultural a través de la actualización de los programas de formación del profesorado especialista en lengua extranjera

y cohesión europeos. A partir de este momento, se empieza a hablar, por ejemplo, del aula como único contexto de aprendizaje de otras lenguas europeas y se apunta a la figura del docente como único responsable del proceso de enseñanza y aprendizaje.

Inspirados por esta descripción de la nueva realidad lingüística y cultural continental y por las intenciones de la política lingüística y educativa europea, creemos necesario plantear una investigación que sirva para proyectar luz sobre dos aspectos esenciales. En primer lugar, será necesario establecer cuáles son esas necesidades y exigencias que la nueva política lingüística europea ha originado. Y, en segundo lugar, será necesario realizar un análisis de un modelo de programa de formación del nuevo profesorado especialista en lengua extranjera concreto para descubrir si se está teniendo éxito en la habilitación del nuevo docente como fuente de conocimiento lingüístico y cultural para el aula de Educación Primaria o si, por el contrario, los esfuerzos realizados desde el marco del Espacio Europeo de Educación Superior no constituyen la respuesta que la nueva Europa multicultural y multilingüe de este siglo XXI requiere.

\section{Palabras clave:}

Competencias; cultura; educación; formación de agentes culturales; multiculturalidad; multilingüismo. the modern foreign language classroom, for example, is perceived to be the only foreign language learning context and the teacher is assigned the task of accepting sole responsibility for the teaching and learning process that takes place in such a constrained environment.

Inspired by this description of the new European linguistic and cultural reality and by the intentions of the European linguistic and educational policy, we are inclined to conduct a research that helps to shed light on two key aspects. Firstly, it is necessary to establish what those needs and demands that the new European language policy has originated. And secondly, an analysis of a specific foreign language teacher training program seems to be of the utmost importance to discover whether this kind of programs are succeeding in enabling student teachers to become a source of linguistic and cultural knowledge in the Primary Education classroom or, on the contrary, all efforts made from within the European Higher Education Area are not the answer the multicultural and multilingual Europe requires at the turn of the century.

\section{Key words:}

Skills; culture; education; cultural agents training; multiculturalism; multilingualism.

\section{Résumé:}

La consolidation de l'Europe comme unité territoriale après la Seconde Guerre mondiale, détermine l'apparition d'un sous entendu multilingue et multiculturelle sans précédent. La politique linguistique du Conseil de l'Europe sera principalement axée sur la garantie de communication, d'enrichissement, de compréhension et de cohésion territoriale et sociale entre les Etats de l'Union. Cet objectif génère un éventail de besoins et exigences auxquels les autorités éducatives devront répondre. Le travail de ces derniers consiste à suivre le chemin entrepris par le Conseil de l'Europe, et ainsi établir des objectifs que les institutions d'enseignement devraient mettre en œuvre pour pouvoir atteindre cette compréhension et cohésion européenne. A compter de ce jour, nous ferons référence à la classe comme étant un lieu contextuel d'apprentissages des langues européennes et à la figure de l'enseignant comme étant le seul responsable du processus d'enseignement et apprentissage.

Inspiré par cette description des nouvelles réalités linguistiques et culturelles continentales, ainsi que de la politique linguistique et éducative européenne, il est nécessaire de proposer une recherche visant à projeter de la lumière sur deux aspects clés. Premièrement, la nouvelle politique européenne de la langue est à l'origine de ces besoins et exi- 
La comunicación, interacción y entendimiento en la Europa multicultural a través de la actualización de los programas de formación del profesorado especialista en lengua extranjera

Gabriel SÁnChez SánChez

gences, dont il est nécessaire de dresser la liste. Deuxièmement, nous devrions effectuer une analyse d'un programme de formation de modèle pour les nouveaux enseignants spécialisés en langue étrangère. En particulier, afin de pouvoir découvrir si cela permet un nouvel enseignement comme source de connaissances linguistiques et culturelles dans la salle de classe en Education primaire. Les efforts réalisés par le cadre de l'Espace européen de l'enseignement supérieur ne sont pas la réponse à la nouvelle Europe multiculturelle et multilingue dont ce siècle a besoin.

\section{Mots clés:}

Compétences; culture; éducation; formation des agents culturels; multiculturalisme; multilinguisme.

Fecha de recepción: 25-3-2015

Fecha de aceptación: 13-4-2015

\section{Introducción}

El proceso de unificación europea debe gran parte de su éxito a una política lingüística del Consejo de Europa que estaba orientada a la búsqueda y una promoción de una comunicación e interacción que favoreciera el entendimiento entre los estados europeos. La labor del Consejo para la Cooperación Cultural, organismo adscrito al Consejo de Europa, está delimitada por tres principios básicos establecidos en esta línea. El primero de ellos hace referencia al ecléctico patrimonio lingüístico y cultural de Europa que constituye un recurso común de indudable validez para la promoción de un enriquecimiento y comprensión mutuos. El segundo de los principios defiende un mayor conocimiento de las lenguas europeas modernas como instrumento con el que se garantice el éxito de la interacción y la comunicación entre ciudadanos de los países miembros y como escudo para luchar contra los prejuicios o la discriminación en que estos acaban derivando. Por último, el tercer principio apuesta por una continuada cooperación y coordinación de las diferentes políticas nacionales en el campo del aprendizaje y la enseñanza de lenguas con el fin de poder alcanzar una mayor convergencia a nivel europeo (Consejo de Europa, 2002: 2), consiguiendo de este modo que la unificación europea sea el objetivo primario de todas y cada una de las políticas educativas comunitarias.

A principios del siglo XXI las Administraciones educativas apuestan por un nuevo proceso de educación obligatoria que combine calidad y equidad como aspectos clave y renovadores de todo el proceso educativo. La Ley Orgánica de Educación (LOE) nace al amparo de una serie 
La comunicación, interacción y entendimiento en la Europa multicultural a través de la actualización de los programas de formación del profesorado especialista en lengua extranjera

Gabriel SánChez SÁnchez

de principios fundamentales entre los que destaca aquel que guarda relación con la necesidad de asegurar una educación de calidad a ciudadanos de ambos sexos con el fin de conseguir que todos ellos logren alcanzar el máximo desarrollo de sus capacidades individuales, sociales, intelectuales, culturales y emocionales (BOE, 2006: 17159). Conscientes de que en ese proceso de desarrollo personal y cognitivo del discente el elemento que va a influir más decisivamente es el propio docente, otro de los principios fundamentales propone un avance hacia la consecución de los objetivos educativos comunes planteados por la Unión Europea que conlleve una mejora progresiva de los programas de habilitación de los docentes.

El presente trabajo de investigación parte de un análisis de los documentos oficiales en que son recogidas las directrices de la política adoptada por las Administraciones educativas. A partir del tipo de información obtenido tras esa revisión, serán propuestos dos objetivos. En primer lugar, como objetivo general, se planteará la necesidad de poner al descubierto las necesidades y exigencias que la nueva política europea en materia de cultura extranjera plantea para la Educación Primaria. Y en segundo lugar, como objetivo más específico, se intentará averiguar si el nuevo profesorado en formación está siendo habilitado para actuar como representante de esa cultura extranjera en el aula y si, de no estarlo, sería necesario plantear una revisión con carácter de urgencia de los programas de formación inicial del profesorado vigentes en el marco del Espacio Europeo de Educación Superior.

\section{Documentos oficiales, objetivos educativos y la figura del docente}

La labor de las Administraciones educativas continúa con las directrices trazadas por el Consejo de Europa en materia de consolidación y buen entendimiento entre los estados europeos y traducen su esfuerzo en el planteamiento de una serie de objetivos educativos que quedan recogidos en todo el abanico de documentos oficiales. En 2006, entre los objetivos establecidos para la etapa de la educación obligatoria básica, destaca el de adquirir en, al menos, una lengua extranjera la Competencia comunicativa básica con la que expresar y comprender mensajes sencillos y desenvolverse en situaciones cotidianas (BOE, 2006: 17168). 
La comunicación, interacción y entendimiento en la Europa multicultural a través de la actualización de los programas de formación del profesorado especialista en lengua extranjera

Gabriel Sánchez Sánchez

En la misma línea de actuación, en otro de los documentos oficiales, el currículo de la Educación Primaria en la Región de Murcia, se insiste en que, entre las habilidades y destrezas que el alumnado ha de haber adquirido al finalizar la etapa mencionada, debe estar incluido el conocimiento de, al menos, una lengua extranjera (BORM, 2007: 26389). Otra de las capacidades a las que se alude también es a la de valorar la lengua extranjera como instrumento para comunicarse oralmente en el aula y como medio de comunicación y entendimiento entre personas de otras culturas europeas (BORM, 2007: 26431-34).

En este mismo documento se pone de manifiesto una realidad social que repercute negativamente sobre el logro de aquellos objetivos educativos planteados desde las Administraciones educativas. Se advierte de que, a pesar de la reciente llegada a nuestro país de ciudadanos de otros países europeos, su presencia es todavía escasa en nuestras sociedades. Precisamente como consecuencia de ello, la situación concreta de la clase de idiomas modernos habrá de empezar a ser percibida como el único contexto donde el alumnado de Primaria puede hacer uso de la lengua extranjera como medio de interacción para la producción y comprensión de mensajes sencillos que constituyen su discurso oral y escrito en las situaciones cotidianas recreadas ad hoc. Además, por otro lado, esa misma realidad social va a condicionar la actuación del profesorado para la Educación Primaria que se va a ver obligado a actuar como modelo de inspiración lingüística (BORM, 2007: 26430).

Sin embargo, concebir este nuevo rol del docente en el proceso educativo para la unificación europea como una entidad monodimensional solo demostraría que se está obviando la estrecha e indisoluble relación entre los conceptos de lengua y cultura. El primero de ellos debe ser considerado como un producto cultural y el segundo alcanza su consolidación a través de la lengua, lo que significa que las culturas manifiestan sus creencias y valores en el habla (Claxton, 2001: 266). Partiendo de este hecho sería posible definir la figura del nuevo docente como una entidad compleja compuesta por una dimensión lingüística y otra dimensión cultural. Esto significa que a su papel como modelo de inspiración lingüística en el aula de idiomas habrá que añadir un rol como modelo de inspiración cultural. 


\section{El nuevo profesorado como modelo de inspiración cultural}

De entre las exigencias de la política lingüística para el entendimiento y la convivencia pacífica del Consejo de Europa deriva la necesidad de conseguir despertar un interés por la figura del docente como representante de la cultura extranjera (BORM, 2007: 26388). Alcanzar ese reconocimiento va a depender de una actuación docente que esté bien sustentada en actitudes y capacidades que el nuevo profesorado haya adquirido previamente durante su formación académica. Del éxito de su actuación como representante de la cultura extranjera van a depender dos de los aspectos más cruciales para alcanzar la comunicación y el entendimiento anhelados para Europa: el nivel de conocimiento de la cultura asociada a la lengua extranjera y la capacidad para adoptar y hacer propios aspectos de aquella. Por un lado, el interés que pueda sentir el alumnado de primaria para adquirir el conocimiento de la cultura extranjera será directamente proporcional al grado de curiosidad que la actuación del docente consiga despertar en él (Durant, 2003: 32) (Tiba, 2009: 165). Por otro lado, un interés cultural considerable está ligado al despertar de una capacidad de imitación con la que el alumnado potencia y da sentido a sus propios actos (Ovejero y Rodríguez, 2005: 41) dentro y fuera del entorno cultural cerrado recreado en el aula de idiomas.

En relación a ese conocimiento y a las capacidades del alumnado, el currículo de la Educación Primaria en la Comunidad Autónoma de la Región de Murcia programa para esta etapa de la educación básica la adquisición de aspectos socioculturales con los que se ha de conformar el conocimiento que promueva el respeto y el interés por las diferentes realidades sociales y culturales (BORM, 2007: 26430). En lo relativo al aprendizaje de la dimensión cultural de la lengua extranjera, al finalizar el segundo ciclo se valorará que el alumnado sea capaz de mostrar interés y curiosidad hacia las personas que hablan la lengua extranjera y que sea capaz de identificar aspectos de la vida cotidiana, costumbres y celebraciones de los países donde la lengua extranjera es el idioma oficial (BORM, 2007: 26434). Al finalizar el tercer ciclo se espera que el alumnado haya afianzado los logros anteriores y demuestre un interés por establecer relaciones personales con las personas que hablan la lengua extranjera con la ayuda de las tecnologías de la información y la comunicación así como de comparar aspectos de la vida cotidiana, costumbres, celebraciones y tradiciones con aspectos, costumbres, 
La comunicación, interacción y entendimiento en la Europa multicultural a través de la actualización de los programas de formación del profesorado especialista en lengua extranjera

Gabriel SánChez SánChez

etc., propios, desarrollando de este modo una conciencia intercultural (BORM, 2007: 26435).

Resulta fácil asociar el logro de estos objetivos a una actuación convincente del docente como modelo de inspiración cultural. Del mismo modo, resulta fácil equiparar el grado de éxito del maestro como representante cultural al nivel de adquisición y desarrollo de un conjunto de habilidades, destrezas y conocimientos que dan fuerza a su actuación y dotan de sentido al contexto lingüístico y cultural recreado en el aula de idiomas. El acceso a esa serie de habilidades depende de la adquisición de una Competencia Intercultural.

\section{El aporte de la Competencia Intercultural al proceso de formación}

Tras un repaso semántico realizado en torno a este concepto, la Competencia Intercultural podría definirse como una habilidad o conjunto de habilidades que deben ser puestas en práctica durante intercambios comunicativos con personas a las que reconocemos, y que se reconocen a sí mismas, como pertenecientes a entornos socioculturales diferentes. Esta condición permite definir a la interacción comunicativa como intercultural y entre las habilidades que son requeridas para que esta se desarrolle con éxito se encuentran una actitud de apertura, una predisposición a hacer frente al sentimiento de incertidumbre que origina esta situación (Alred, 2003: 24) y la capacidad para abandonar la seguridad que confiere el punto de vista propio (Byram, 2005: 26), para desenvolverse en el contexto intercultural (Aguado, 2003: 141), y para interactuar apropiadamente con esos otros representantes culturales (Guilherme 2002: 297).

Michael Byram y Geneviève Zarate (1994) construyen un modelo de Competencia Intercultural en torno a unas habilidades de interpretación y relación ('savoir comprendre'), habilidades de descubrimiento e interacción ('savoir apprendre/faire'), una conciencia cultural crítica ('savoir s'engager'), unas actitudes ('savoir être') y un conocimiento ('savoirs') (Byram, 1997: 49-54).

Las habilidades de interpretación y relación tienen que ver con la capacidad para interpretar un documento o suceso propio de la cultura meta y ponerlo en relación con sucesos o documentos de la cultura materna. 
La comunicación, interacción y entendimiento en la Europa multicultural a través de la actualización de los programas de formación del profesorado especialista en lengua extranjera

Gabriel SánChez SÁnchez

Las habilidades de descubrimiento e interacción se relacionan con la capacidad para adquirir nuevo conocimiento sobre el conjunto de la sociedad extranjera, sobre sus productos y modos de actuar culturales y la capacidad de manejar conocimiento, actitudes y habilidades bajo las restricciones de una comunicación e interacción a tiempo real.

La conciencia cultural crítica es la habilidad para evaluar, de manera crítica y desde principios explícitos, perspectivas, modos de actuar y productos en la propia cultura y en la cultura y país de la lengua extranjera.

Las actitudes incluyen la curiosidad y la apertura, la predisposición a suspender su descrédito hacia la cultura extranjera así como el crédito hacia la propia, todos ellos resultado del deseo personal del futuro docente.

El conocimiento incluido en la Competencia Intercultural aglutina información general del país en el que la lengua extranjera es el idioma oficial además de información más específica en torno a la sociedad y la cultura con las que se relaciona a esa lengua extranjera. Este complejo corpus de información puede ser adquirido desde tres posibles fuentes:

- Una fuente primaria (familiar) provee al individuo con información que proviene de experiencias personales o creencias populares de familiares, en primera instancia, y amigos o conocidos, en segunda. La veracidad (y utilidad en la elaboración del conocimiento intercultural) de este tipo de información es bastante dudosa aún cuando se trate de experiencias personales pues cabe la posibilidad de que estas ofrezcan al individuo una información subjetiva.

- Una fuente secundaria (didáctica) como pueden ser los libros de texto, las enciclopedias, etc. A pesar del uso cotidiano de este tipo de información en el ámbito docente, su veracidad debe ser igualmente cuestionada puesto que esta puede responder a intereses de las editoriales. La aportación y formación de docentes y otros profesionales es esencial para evitar la transmisión de información distorsionada e irreal.

- Una fuente terciaria (mediática) ofrece información a través de medios de comunicación diversos (i.e. televisión, prensa escrita, Internet, etc.). Debido a la enorme e indiscutible influencia que ejercen los medios de comunicación en las masas, su veracidad debe ser cuestionada en todo momento ante la posibilidad de que pudiera responder a cualquier tipo de consigna ideológica. 
La comunicación, interacción y entendimiento en la Europa multicultural a través de la actualización de los programas de formación del profesorado especialista en lengua extranjera

Gabriel SánChez SánChez

\section{El conocimiento específico como elemento diferenciador}

Como hemos visto, desde las Administraciones educativas se apuesta por la dimensión del docente como modelo de inspiración lingüística. A partir de la estrecha relación entre lenguaje y cultura y las implicaciones de la escasa presencia de hablantes nativos de la lengua extranjera en nuestro país, se proponía ampliar el perfil del docente añadiendo a la mencionada, una dimensión como figura de inspiración cultural. Alcanzar ese estatus va a requerir de un conocimiento específico que permita al aspirante convertirse en representante de la cultura extranjera en el aula de primaria. El conocimiento asociado a la Competencia Intercultural es el resultado de la combinación de dos tipos de información. Por un lado, información factual del país o países donde se habla la lengua extranjera en relación, por ejemplo, a las principales características geográficas, medioambientales, demográficas, económicas, políticas, etc. Toda esa información constituye el conocimiento del mundo. Y, por otro lado, un tipo de información directamente relacionada con las características distintivas de la sociedad y cultura europea en donde la lengua extranjera es el idioma oficial. Este otro tipo de información es conocida como conocimiento sociocultural (Consejo de Europa, 2002: 100). Tradicionalmente se ha hecho referencia a una Cultura con $\mathrm{C}$ mayúscula y otra cultura con c minúscula. La primera de ellas estaría ligada al conocimiento del mundo y englobaría, además de toda la información factual mencionada, aquella información relacionada con la historia, las artes, los grandes logros de una comunidad, etc. La segunda de ellas, la cultura con c minúscula, hace referencia a las costumbres, tradiciones y formas de vida de sociedad y cultura extranjera por lo que está más ligada al conocimiento sociocultural. La adquisición de este tipo concreto de información sobre la sociedad y la cultura asociadas a la lengua extranjera se produce mediante un proceso de aprendizaje consciente (Durant, 2003: 20) también conocido como aculturación.

Formado etimológicamente por la preposición latina ' $a d$ ', que significa unión, contacto o cercanía, y 'cultural' en el sentido de conjunto de las realizaciones y formas de vida de un grupo humano, el concepto de aculturación debe ser entendido como contacto intercultural (Juliano, 1993: 5). Para entender el proceso de aculturación de la información sobre la sociedad y la cultura extranjera basta con revisar el proceso de adquisición lingüística. Durante su aprendizaje, El bebé imita los so- 
La comunicación, interacción y entendimiento en la Europa multicultural a través de la actualización de los programas de formación del profesorado especialista en lengua extranjera

Gabriel SánChez SÁnchez

nidos que escucha en el habla de la madre. Mediante la imitación, va adquiriendo el reducido número de unidades de sonido de su lengua materna. Cada una de esas unidades va siendo ordenada en un número fijo de casillas en su mente. Con el tiempo y con el uso continuado, estas casillas de sonido se van afianzando y fosilizando en su mente. Este proceso se repite en cada lengua con la salvedad de que cada lengua cuenta con un número diferente de sonidos encasillados $\left(\mathrm{O}^{\prime} \mathrm{Connor}\right.$, 1980: 2). De un modo similar, la adquisición del conocimiento sociocultural o cultura con c minúscula va a obligar al individuo a clasificar la nueva información en siete casillas que representan las características distintivas de cualquier sociedad europea y que habrá ido generando a lo largo de su vida en su experiencia en su entorno sociocultural materno. Las diferentes casillas son las siguientes: 1) La vida diaria: comida y bebida, horarios, modales; días festivos; el mundo laboral; y actividades de ocio. 2) Las condiciones de vida: niveles de vida; condiciones de la vivienda; medidas de asistencia social. 3) Las relaciones personales: estructura social y relaciones familiares; relaciones entre sexos, entre generaciones, en situaciones de trabajo, con la autoridad, de raza y comunidad y entre grupos políticos y religiosos. 4) Los valores, creencias y actitudes con respecto a: la clase social, culturas regionales, identidad nacional, países extranjeros, etc. 5) El lenguaje corporal: conocimiento de las convenciones que rigen dicho comportamiento. 6) Las convenciones sociales: puntualidad, regalos, ropa, convenciones y tabúes referidos al comportamiento y a las conversaciones, despedida. Y, por último, 7) el comportamiento ritual en: ceremonias religiosas, representaciones y ceremonias públicas, celebraciones, festividades, etc. (Consejo de Europa, 2002: 100-101).

Durante el proceso de aculturación, el individuo entrará en contacto con nueva información sobre la sociedad y cultura extranjera. Este conocimiento sociocultural contribuye a crear en los seres humanos un sentimiento de grupo que comparte rituales, normas, valores, creencias, pautas de comportamiento cuya aceptación y puesta en práctica favorece y potencia la cohesión del grupo (Bueno, 1995: 362). De la adquisición de ese conocimiento sociocultural dependerá la integración personal del individuo en la sociedad y cultura extranjeras (Aguirre, 1993: 220). Es ese sentimiento de grupo y de pertenencia al mismo el que da sentido a la existencia humana y el que distingue a cada ser humano como miembro de un grupo sociocultural concreto y definido. Precisamente por ello, 
La comunicación, interacción y entendimiento en la Europa multicultural a través de la actualización de los programas de formación del profesorado especialista en lengua extranjera

va a ser aquella cultura con c minúscula o conocimiento sociocultural la información clave de la que debe empaparse cualquier programa de formación que aspire a formar al nuevo profesorado de Educación Primaria como representante de la cultura extranjera o modelo de inspiración cultural. Descubrir si al nuevo profesorado especialista en lengua extranjera le ha sido facilitada la adquisición de este conocimiento sociocultural es uno de los objetivos específicos con los que partía el presente trabajo de investigación.

\section{Trabajo de campo}

Con el propósito de descubrir si la adquisición del conocimiento sociocultural era objetivo reconocido de los programas de formación, se plantea analizar la diplomatura de Magisterio como modelo de programa de formación del nuevo profesorado de Educación Primaria para descubrir si este responde con éxito a las necesidades y exigencias de la realidad multicultural (y multilingüe) europea de este siglo XXI, es decir, si el nuevo profesorado está siendo formado para actuar como modelo de inspiración cultural o representante de la cultura extranjera en el aula de primaria. Con este fin, se lleva a cabo un estudio con un total de cien estudiantes que cursaban los estudios conducentes al título de MaestroEspecialidad en Lenguas Extranjeras (Inglés) en la Facultad de Educación de la Universidad de Murcia. Fueron seleccionados los treinta y dos estudiantes que cursaban el primer curso, los treinta y cinco de segundo curso y los treinta y tres de tercer y último curso. La investigación es, por tanto, un estudio diacrónico de la Competencia Intercultural con sujetos de muestra representantes de cada uno de los tres cursos académicos de que constaba la diplomatura de Magisterio.

El desarrollo de la investigación es programado en las siguiente fases: 1) elaboración del instrumento de medida; 2) recogida de información; 3) análisis, categorización, organización e interpretación de los datos recogidos.

Para la elaboración del instrumento de recogida de información, se tuvo en cuenta el hecho de que la información requerida (i.e. conocimiento sociocultural) no es uno de los elementos más fácilmente observables de la Competencia Intercultural en el contexto en el que se iba a desarrollar la investigación (i.e. aula universitaria). Se optó entonces 
por la elaboración de un instrumento de medida o Prueba de Escenarios Prácticos que permitiera a los estudiantes encuestados expresar sus ideas y modos de actuar personales en la resolución de la problemática planteada en torno a la interacción comunicativa intercultural presentada en siete situaciones hipotéticas pero posibles en la vida real.

Durante la segunda fase se procedió a la recogida de información que, debido a las obligaciones académicas del estudiantado, se realiza en una única sesión de sesenta minutos con cada grupo, realizándose un total de tres sesiones. A los individuos encuestados se les pide que lean atentamente los siete escenarios prácticos, que presten atención a la problemática planteada y que contesten siguiendo los dictados de su propio conocimiento, intuición y buen juicio.

Por último, en la tercera fase de la investigación se procede a analizar cualitativamente, categorizar, organizar e interpretar los datos obtenidos en los discursos escritos ofrecidos por los sujetos de muestra con el fin de intentar hallar muestras del conocimiento sociocultural (cultura con c minúscula) necesario del que depende, además de los elementos listados, un buen dominio de la Competencia Intercultural y, como consecuencia, una actuación eficaz y convincente como representante de la cultura extranjera en el aula.

\section{Resultados}

La información ofrecida por los treinta y dos sujetos de muestra de primer curso aparece recogida en la tabla 1 . De las doscientas veinticuatro respuestas ofrecidas, tan solo veintinueve de ellas (12.9\%) son reflejo de un elevado grado de conocimiento sociocultural frente a las ciento catorce respuestas $(50.8 \%)$ que delatan un desconocimiento sociocultural o las ochenta y una (36.1\%) que delatan carencias cognitivas. 
La comunicación, interacción y entendimiento en la Europa multicultural a través de la actualización de los programas de formación del profesorado especialista en lengua extranjera

Gabriel SánChez SánChez

Tabla 1. Información específica sobre el conocimiento sociocultural de los sujetos del grupo de primer curso.

\begin{tabular}{lccc} 
& \multicolumn{3}{c}{ TABLA GENERAL sobre Competencia Intercultural } \\
\cline { 2 - 4 } & \multicolumn{3}{c}{ Respuestas y porcentajes } \\
\cline { 2 - 4 } & BIEN & MAL & CARENCIAS \\
\hline Caso 1 & $6(18.7 \%)$ & 0 & $26(81.2 \%)$ \\
\hline Caso 2 & $10(31.2 \%)$ & $21(65.6 \%)$ & $1(3.1 \%)$ \\
\hline Caso 3 & $1(3.1 \%)$ & $13(40.6 \%)$ & $18(56.2 \%)$ \\
\hline Caso 4 & $8(25 \%)$ & $9(28.1 \%)$ & $15(46.8 \%)$ \\
\hline Caso 5 & $4(12.5 \%)$ & $26(81.2 \%)$ & $2(6.2 \%)$ \\
\hline Caso 6 & 0 & $23(71.8 \%)$ & $9(28.1 \%)$ \\
\hline Caso 7 & 0 & $22(68.7 \%)$ & $10(31.2 \%)$ \\
\hline TOTAL respuestas & $29(12.9 \%)$ & $114(50.8 \%)$ & $81(36.1 \%)$ \\
\hline
\end{tabular}

El análisis de los datos arrojados por los treinta y cinco sujetos de muestra de segundo curso evidencia un descenso en el número de respuestas apropiadas y un aumento de respuestas mal o con carencias con respecto a los resultados del grupo del curso anterior. En la tabla 2, se aprecia cómo, de las doscientas cuarenta y cinco respuestas analizadas, sólo veintitrés de ellas (9.3\%) reflejan un buen conocimiento sociocultural. En ciento treinta respuestas se distingue un considerablemente negativo desconocimiento sociocultural (53\%) y en noventa y dos $(37.5 \%)$, carencias en ese conocimiento de la cultura extranjera.

Tabla 2. Información específica sobre el conocimiento sociocultural de los sujetos del grupo de segundo curso.

\begin{tabular}{lccc} 
& \multicolumn{3}{c}{ TABLA GENERAL sobre Competencia Intercultural } \\
\cline { 2 - 4 } & \multicolumn{3}{c}{ Respuestas y porcentajes } \\
\cline { 2 - 4 } & BIEN & MAL & CARENCIAS \\
\hline Caso 1 & $1(2.8 \%)$ & $1(2.8 \%)$ & $33(94.2 \%)$ \\
\hline Caso 2 & $6(17.1 \%)$ & $23(65.7 \%)$ & $6(17.1 \%)$ \\
\hline Caso 3 & 0 & $9(25.7 \%)$ & $26(74.2 \%)$ \\
\hline Caso 4 & $15(42.8 \%)$ & $5(14.2 \%)$ & $15(42.8 \%)$ \\
\hline Caso 5 & $1(2.8 \%)$ & $34(97.1 \%)$ & 0 \\
\hline Caso 6 & 0 & $31(88.5 \%)$ & $4(11.4 \%)$ \\
\hline Caso 7 & 0 & $27(77.1 \%)$ & $8(22.8 \%)$ \\
\hline TOTAL respuestas & $23(9.3 \%)$ & $130(53 \%)$ & $92(37.5 \%)$ \\
\hline
\end{tabular}


La comunicación, interacción y entendimiento en la Europa multicultural a través de la actualización de los programas de formación del profesorado especialista en lengua extranjera

Gabriel SánChez SÁnchez

Finalmente, de las doscientas treinta y una respuestas ofrecidas por los treinta y tres sujetos de muestra de tercer curso, treinta y siete (16\%) evidencian un buen conocimiento sociocultural, ciento nueve respuestas $(47.1 \%)$, un desconocimiento de la cultura extranjera y ochenta y cinco $(36.7 \%)$ responden a discursos escritos con importantes carencias cognitivas (tabla 3).

Tabla 3. Información específica sobre el conocimiento sociocultural de los sujetos del grupo de tercer curso.

\begin{tabular}{lccc} 
& \multicolumn{3}{c}{ TABLA GENERAL sobre Competencia Intercultural } \\
\cline { 2 - 4 } & \multicolumn{3}{c}{ Respuestas y porcentajes } \\
\cline { 2 - 4 } & BIEN & MAL & CARENCIAS \\
\hline Caso 1 & 0 & 0 & $33(100 \%)$ \\
\hline Caso 2 & $11(33.3 \%)$ & $15(45.4 \%)$ & $7(21.2 \%)$ \\
\hline Caso 3 & 0 & $12(36.3 \%)$ & $21(63.6 \%)$ \\
\hline Caso 4 & $22(66.6 \%)$ & $2(6 \%)$ & $9(27.2 \%)$ \\
\hline Caso 5 & 0 & $30(90.9 \%)$ & $3(9 \%)$ \\
\hline Caso 6 & $1(3 \%)$ & $27(81.6 \%)$ & $5(15.1 \%)$ \\
\hline Caso 7 & $3(9 \%)$ & $23(69.6 \%)$ & $7(21.2 \%)$ \\
\hline TOTAL respuestas & $37(16 \%)$ & $109(47.1 \%)$ & $85(36.7 \%)$ \\
\hline
\end{tabular}

A pesar haber quedado demostrado un evidente e ínfimo desconocimiento de la cultura extranjera por parte del nuevo profesorado especialista en lengua extranjera en formación, es importante saber apreciar los datos positivos en cuanto al grado de conocimiento sociocultural o cultura con c minúscula porque resultaría esperanzador hasta cierto punto pensar que quizás esas treinta y siete respuestas correctas (16\%) vienen avaladas por cierto grado de conocimiento de la cultura extranjera que podría haber sido adquirido por los estudiantes gracias a la asignatura de 'Cultura y Civilización de la Lengua Extranjera', asignatura obligatoria de tercer curso y la única de la diplomatura enfocada a la enseñanza de aspectos socioculturales relacionados con la lengua extranjera con la que se pretendía despertar el interés de los futuros docentes por la cuestión intercultural mediante, por ejemplo, la elaboración de materiales.

\section{Conclusiones}

En una representación simplificada de la dinámica en el aula, el maestro enseña y el alumno aprende, el maestro rezuma todo el conocimiento 
La comunicación, interacción y entendimiento en la Europa multicultural a través de la actualización de los programas de formación del profesorado especialista en lengua

y el alumno lo absorbe. A pesar de haber sido revisitado y actualizado, este modelo de interacción representa con precisión el tipo de relación que se defiende en estas páginas entre un docente-modelo y un discenteimitador y respalda la creencia de que todo conocimiento sociocultural que no provenga de un profesorado comprometido con su tarea puede estar distorsionado y contribuir a la generación de prejuicios y la perpetuación de estereotipos por parte de un alumnado desconocedor de otras realidades culturales. La capacidad del nuevo docente para actuar como representante de la cultura extranjera ha de ser considerada como directamente proporcional al grado de conocimiento sociocultural ('savoir') que posea este y al de su habilidad para personificarlo ('savoir être').

Con el presente estudio se han recabado datos que conducen a conclusiones obvias en relación a los dos objetivos planteados para el presente trabajo de investigación. En lo que concierne a la habilitación del nuevo profesorado en formación como figura de inspiración cultural, los datos obtenidos en torno a la Competencia Intercultural delatan en los sujetos de muestra un escaso conocimiento sociocultural, lo que va a malograr su actuación como representante de la cultura extranjera en el aula según lo estipula la ley orgánica de educación. Un docente que no haya adquirido este tipo de conocimiento desconoce los elementos clave que confieren a cada los hablantes nativos de la lengua extranjera el sentimiento de grupo cultural y, por tanto, no habrá experimentado el sentimiento de pertenencia al mismo.

Por otra parte, la ausencia de un modelo de inspiración cultural constituye un impedimento a la hora de conseguir que el alumnado de Educación primaria muestre respeto por una serie de valores, creencias, pautas de comportamiento, etc., (cultura con c minúscula) que le son ajenos y que pueden llegar a producirle cierto grado de extrañeza. A partir de este instante, ese alumnado carente de un referente cultural con fundamento entra en una espiral descendiente en la que se verán socavados su interés y curiosidad por la cultura extranjera y los miembros de esta y, finalmente, su capacidad innata de imitación. Este desalentador desenlace va a repercutir negativamente sobre el objetivo de la política lingüística del Consejo de Europa de garantizar la comunicación, la interacción y el entendimiento entre los ciudadanos de los estados de la unión.

Las conclusiones alcanzadas con la investigación realizada revelan que uno de los puntos débiles más alarmantes de la extinta diplomatura 
La comunicación, interacción y entendimiento en la Europa multicultural a través de la actualización de los programas de formación del profesorado especialista en lengua extranjera

de Magisterio Lengua Extranjera (Inglés) como modelo de programa de formación del profesorado era el escaso éxito logrado en la formación de docentes especialistas en la cultura extranjera, lo que comprometía el logro de los objetivos educativos propuestos por el Consejo de Europa en relación a la mejora en la calidad y eficacia de la formación cultural de la figura del docente y justificaría una revisión con carácter de urgencia de los programas de formación inicial de profesorado que se encuentran actualmente vigentes en el marco del Espacio Europeo de Educación Superior.

\section{Referencias bibliográficas}

AGUADO ODINA, T. (2003). Pedagogía intercultural. Madrid: McGraw-Hill.

AGUIRRE, A. (1993). Cultura. En A. Aguirre Baztán (Ed.), DiccionarioTemático de Antropología. 2a Edición. Colección "Historia de la Antropología Española", n³ (pp. 152-159). Barcelona: Macombo, S.A.

ALRED, G. (2003). Becoming a 'Better Stranger': A Therapeutic Perspective on Intercultural Experience and/as Education. En G. Alred, M. Byram y M. Fleming (Eds.). Intercultural Experience and Education (pp.14-30). Clevedon: Multilingual Matters Ltd.

BERNABÉ VILLODRE, M. (2014). Propuestas didácticas musicales para trabajar interculturalmente en ESO: la música pop de mestizaje. Espiral. Cuadernos del profesorado, 7(13), 59-70.

Boletín Oficial del Estado. (2014). Disposiciones Generales. Número 206. Sábado, 6 de septiembre de 2014, pp.33230-33287.

Boletín Oficial del Estado. (2006). Disposiciones Generales. Número 106. Jueves, 4 de mayo de 2006, pp. 17158-17207.

Boletín Oficial de la Región de Murcia. (2007). Disposiciones Generales. Número 211. Miércoles, 12 de septiembre de 2007, pp. 26387-26450.

BUENO, A. (1995). Sociolinguistic and Sociocultural Competence. En N. McLaren y D. Madrid. A Handbook for TEFL (pp. 345-374). Alcoy, Marfil. (citado en Trujillo 2006, Cultura, comunicación y lenguaje, p.36).

BYRAM, M. (1997). Teaching and assessing intercultural communicative competence. Clevedon: Multilingual Matters.

BYRAM, M. (2005). Intercultural competence and the politics of foreign language education. En R. Luengo, G. De la Maya y P. Gutiérrez (Eds.). Cultura, Interculturalidad y Didáctica de la lengua y la literatura (pp. 25-34). Badajoz: Universidad de Extremadura/SEDLL.

BYRAM, M. y ZARATE, G. (1994). Definitions, Objectives and Assessment of Socio-cultural Competence. Paper for the Council of Europe Education Commitee, Strasbourg, Mimeo.

CLAXTON, G. (2001). Aprender: el reto del aprendizaje continuo. Barcelona: Paidós. 
La comunicación, interacción y entendimiento en la Europa multicultural a través de la actualización de los programas de formación del profesorado especialista en lengua extranjera

Gabriel Sánchez Sánchez

Consejo de Europa. (2002). Marco Común Europeo de Referencia para Las Lenguas. Recuperado el 13 de marzo de 2015, de http://cvc.cervantes.es/obref/marco/

DURANT, A. (2003) ( $2^{\mathrm{a}}$ ed.). Facts and Meanings in British Cultural Studies. En S. Bassnett (ed.). Studying British Cultures (pp. 20-40). London: Routledge.

GUILHERME, M. (2002). Intercultural Competence. En M. Byram y C. Brumfit. The Routledge Encyclopedia of Language Teaching and Learning (pp.297-300). London: Routledge.

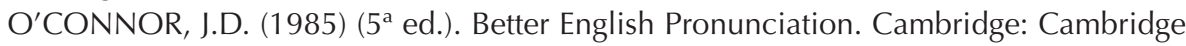
University Press.

OVEJERO BERNAL, A. y RODRÍGUEZ, F.J. (Coords.) (2005). La convivencia sin violencia. Alcalá de Guadaira (Sevilla): MAD.

PARICIO TATO, Silvina. (2014). Competencia intercultural en la enseñanza de lenguas extranjeras. Porta Linguarum: revista internacional de didáctica de las lenguas extranjeras, 21, 215-226.

TIBA, I. (2009). Quien ama educa. Madrid: Aguilar.

VEZ, J.M. y GONZÁLEZ PIÑEIRO, M. (2014). Intercultural competence and the European dimension. En D. MADRID FERNÁNDEZ y N. MCLAREN (Coords.) TELF in primary education (pp. 342-382). Granada: Universidad de Granada.

WENINGER, C. y ZOLTÁN KISS, T. (2013). Culture in English as a Foreign Language (EFL) Textbooks: A Semiotic Approach. TESOL quarterly: a journal for teachers of Englsih to speakers of other languages and of standard English as a second dialect, 47(4), 694-716.

ZAPATA ROS, M. (2014). Hacia una nueva interculturalidad (educativa). RED: Revista de Educación a Distancia, 41, 1-20. 
